\title{
Factores que afectan el proceso de vigilancia epidemiológica de la mortalidad materna en el departamento del Cauca
}

\author{
Mario Delgado Noguera*; Carmen Daza Córdoba** \\ Recibido: Febrero 10 I 2000 \\ Revisado: Marzo 15 / 2000 \\ Aceptado: Junio 9 I 2000
}

\section{RESUMEN}

ANTECEDENTES: En el departamento del Cauca la tasa de mortalidad materna se ha incrementado. En el periodo 1991-1996 ha pasado de 1.5 a 2.3 muertes por 1000 nacidos vivos. Esta tasa se sitúa por encima de la nacional. La vigilancia en salud pública se ha visto afectada por el cambio de régimen de seguridad social.

DISEÑO: Investigación cualitativa por medio de grupos locales

CONTEXTO: Grupos encargados de la vigilancia epidemiológica de la mortalidad materna en hospitales locales de 14 municipios del departamento del Cauca

PARTICIPANTES: 14 grupos de Vigilancia reunidos en 4 grupos locales, muestreados por conveniencia

PRINCIPALES MEDICIONES: Los grupos de vigilancia se reunieron en 4 grupos locales agrupados por vecindad geográfica y cultural por medio de grabación de audio y video. El análisis se realizó por medio de las técnicas de identificación de temas y densidad de incidencia.

RESULTADOS: Los principales factores identificados por los grupos: Falta de continuidad del personal a cargo, Influencia negativa del proceso de aplicación de la nueva ley de seguridad social, Registro de datos de la mortalidad materna sin análisis, déficit de un sistema estructurado de Vigilancia en salud pública.

CONCLUSIONES: Los grupos locales permitieron identificar debilidades en la aplicación de una política estructurada de la dirección departamental de salud con respecto a la vigilancia de la mortalidad materna. Los intentos de aplicación de una política y un sistema de información en este aspecto han encontrado dificultades por la implementación del nuevo régimen de seguridad social en Colombia.

PALABRAS CLAVES: mortalidad materna, vigilancia epidemiológica, estudios cualitativos, grupos locales.

\section{ABSTRACT}

BACKGROUND: in the last 5 years has been detected an increase in mother mortality in the Cauca department.

OBJETIVE: To determine the affecting factors in the epidemiological surveilIance of mother mortality.

DESING: Cualitative research by means of local groups.

SETTING: Teams in charge of the mother mortality surveilIance in local hospitals of Cauca department.

PARTICIPANTS: 14 surveilIance groups gathered as local groups. Sample method: by convienence.

MAIN OUTCOME MEASURES: The surveilIance groups were gathered by the geographic and cultural vecinity looking for homogeneity in 4 groups. The average number has 12, discussion average time was 90 minutes. Discussion was recorded in video and audio tape. Analysis was done by means or subject identification and incidence density.

RESULTS: the main identified factors were: Lack of stalI continuty, negative influence of de-centralization process. Data register without analysis. Lack of a structured system of surveilIance.

CONCLUSIONS: Focal groups were able to identified lack of a structured policy in the departamental health directions respect to mother mortality. The attempts to establish a policy and a information system had faced difficulties in the new health system in Colombia.

KEY WORDS: maternal mortality, epidemiologic surveilIance, cualitative studies, focus groups.

\footnotetext{
* Profesor asistente, Departamento de Pediatría y Unidad de Epidemiología Clínica, facultad Ciencia de la Salud, Universidad del Cauca.

** Profesor asistente, Departamento de Salud Familiar y Medicina Preventiva, Facultad Ciencias de la Salud, Universidad del Cauca.
} 


\section{Introducción}

La muerte de una mujer por motivo de la maternidad debe ser objeto de preocupación entre los agentes de salud, por su trascendencia social y familiar, porque es un indicador de calidad de salud y de la vida y sobretodo porque la mayoría de las defunciones maternas se originan por complicaciones obstétricas evitables por medio de intervenciones médicas oportunas (1).

La reducción de la mortalidad materna fue acordada por todos los gobiernos de la región de las Américas, durante la XXIII Conferencia Sanitaria Panamericana en 1990, cuando se comprometieron a seguir los lineamientos y estrategias establecidas en el plan de acción de reducción de la mortalidad materna, propuesto por la O.P.S. Este plan tiene como objetivo intervenir las causas directas e indirectas de muerte materna mediante acciones destinadas a mejorar las condiciones de vida de mujeres en edad fértil y la prestación de servicios de salud (2).

En América Latina y el Caribe la probabilidad de que una mujer muera por causas maternas durante su vida reproductiva es de 1 en 79 y el riesgo de morir durante el embarazo y el parto llega a ser más de 100 veces mayor en algunos países en desarrollo que en los desarrollados. El impacto del contexto socioeconómico sobre la mortalidad materna se expresa nítidamente en las diferencias entre países con diferente grado de desarrollo. Así, mientras en Bolivia la probabilidad de que una mujer muera por causas maternas es de 1 en 50, en Canadá es de 1 en 13.000. En Colombia es de 1 en 290, colocándose en una posición intermedia entre los países de la región. Con relación ala tasa de mortalidad materna por 1000 nacidos vivos, Colombia se sitúa, entre los latinoamericanos, en el grupo de países de tasas altas entre 0.5 y 1.4 por 1000 nacidos vivos (2).

La mayor parte de las muertes que se producen por causas obstétricas directas, resultan de complicaciones del estado de embarazo mismo, del parto o puerperio o de intervenciones $\mathrm{u}$ omisiones en su manejo. El aborto, la toxemia, hemorragias, embolia pulmonar y la sepsis puerperal, son las causas directas de las muertes de este grupo (2). Otros factores biosicosociales se han relacionado con la mortalidad materna factores como: No recibir atención en el primer lugar donde se solicitó, madre solterismo, Predisposición no favorable hacia la planificación familiar, Inicio tardío del control prenatal $y$ falta de seguridad social (3) .

En Octubre de 1992 se promueve en Colombia la creación de los sistemas de vigilancia epidemiológica aplicados a la reducción de la mortalidad materna y perinatal $(4,5)$. Este sistema realiza la captación, notificación y análisis de estas muertes, permite explicar su dinámica y planificar la prestación de los servicios de manera que se dirijan a intervenciones eficientes y oportunas $(5,6)$. En el departamento del Cauca, se adoptó a partir de 1993 y ese mismo año los municipios inician la recolección y notificación de información para ser analizada por los Comités de Vigilancia epidemiológica departamental (COVE) (7).

A partir de 1995 la Dirección departamental de Salud en convenio con el Fondo de las Naciones Unidas para la Infancia, adelanta un proyecto a largo plazo para reducir la mortalidad materna y perinatal en el Cauca. En este departamento la tasa de mortalidad materna se ha incrementado como puede observarse en la figura 1. En el período 1991-1996 la mortalidad materna ha pasado de 1.5 a 2.3 muertes por 1000 nacidos vivos. Esta tasase sitúa por encima de la nacional (7). Para este trabajo se han elegido como población en estudio los grupos encargados de los programas de vigilancia porque en ellos se centran no solo los problemas que se originan en el funcionamiento de los servicios de salud, sino los derivados del acceso y de las creencias, actitudes y prácticas sobre las situaciones de salud que rodean el embarazo y el parto por parte de la población.

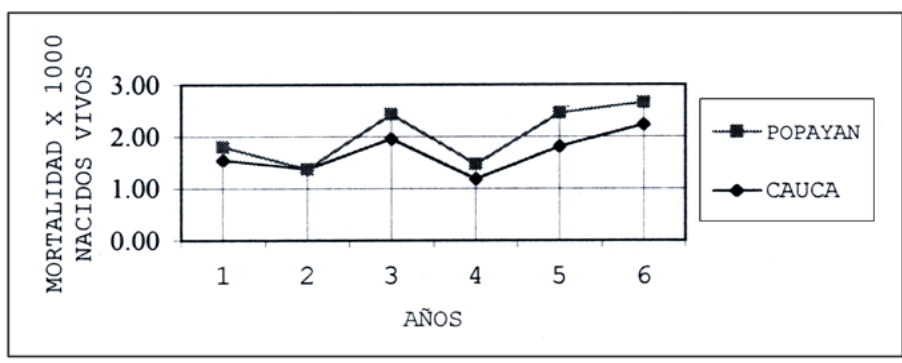

Figura 009i01

\section{TASA DE MORTALIDAD MATERNA EN POPAYAN Y EL CAUCA X 1000 NACIDOS VIVOS, 1991-1996}

La técnica cualitativa de grupos focales tiene como objetivo comprender cuáles son las razones y motivaciones subyacentes a actitudes, preferencias o comportamientos de las personas e intenta recuperar el contexto y las dimensiones humanas en investigación en salud, tratando de suplir las debilidades del enfoque externo, deductivo y experimental de la investigación cuantitativa $(8,9,10,11)$. El grupo focal es una discusión en la cual un pequeño grupo de personas conversa espontáneamente sobre temas considerados importantes para una investigación guiados por un facilitador. Permite a partir de la observación de hechos particulares generar datos válidos, variados, ricos en detalles y establecer hipótesis $(8,9,11)$.

El objetivo del presente trabajo fue explorar aspectos relacionados con la operacionalización del programa de Vigilancia epidemiológica de la mortalidad materna y perinatal en catorce municipios caucanos que hacían parte del convenio Dirección Departamental de Salud - Naciones Unidas. Por otra parte se deseaba identificar fortalezas y debilidades de los comités municipales responsables de esta actividad con el fin de tener elementos para intervenir en los puntos críticos encontrados y optimizar así la estrategia de la vigilancia.

\section{Material y métodos}

Entre Octubre y Diciembre de 1997, previa solicitud de análisis sobre las tendencias de la mortalidad materna y perinatal del periodo de 1985 -1996 a cada uno de los 14 municipios participantes, mediante la técnica cualitativa de grupos focales se exploraron las razones, motivaciones, actitudes, preferencias y comportamientos de las personas encargadas de la vigilancia de la mortalidad 
materna y perinatal. El equipo de investigación lo conformaron 4 personas, dos investigadores principales (médico y enfermera) quienes actuaron siempre como facilitadores y otras dos personas que actuaron cada una como observador o escribiente.

Por conveniencia y reunidos sobre la base de vecindad geográfica, característica que tenía la ventaja de integrar también afinidades culturales y demográficas se conformaron cuatro grupos focales en los municipios cabecera de las antiguas regionales de la dirección departamental así: Popayán, Inzá, Caloto y el Bordo.

Cada grupo fue constituido en promedio por 12 personas, representantes de las instituciones de salud de la zona, participaron hombres y mujeres de diferentes profesiones y cargos, médicos, enfermeras, bacteriólogos, auxiliares de enfermería y estadística. Es importante anotar que no en todos los casos el director acudió a la convocatoria y en ningún equipo el odontólogo estuvo presente. El grado de participación y de relaciones entre funcionarios fue bueno, el nivel de involucramiento del facilitador fue alto y hubo personas dominantes; las salas de reuniones de hospitales y auditorio del Servicio de Salud sirvieron de escenarios de las discusiones las cuales duraron en promedio 90 minutos y se basaron en una serie de preguntas exploratorias derivadas de fa pregunta principal de investigación: ¿¿Cuáles son los factores que afectan el proceso de Vigilancia epidemiológica de la mortalidad materna y perinatal en su municipio?".

Las discusiones se grabaron en su totalidad en audio y una en video; la transcripción se hizo mediante procesador de texto con personal independiente. Las técnicas de análisis aplicadas fueron identificación de temas y densidad de incidencia $(8,11$, 12) y los aspectos explorados se dividieron en factores institucionales (Condiciones y situaciones políticoadministrativas en las cuales se hace posible entender la situación general del programa de vigilancia) y poblacionales (Aspectos culturales, económicos, geográficos, expresados por los participantes que influyen en el control de las muertes perinatales y maternas).

La revisión teórica incluyó la búsqueda de tres temáticas principales: Operacionalización de la Vigilancia Epidemiológica, epidemiología de la mortalidad materna y perinatal y trabajos previos sobre investigación cualitativa aplicada a la Vigilancia Epidemiológica de la mortalidad materna y perinatal. Se realizó búsqueda bibliográfica en MEDLINE entre 1990 y 1998 sin encontrar artículos originales específicos sobre este último tema.

\section{Resultados y discusión}

1. Identificación de temas

\section{Factores institucionales}

- Una política institucional centralizada que no favorecía la autonomía administrativa y trae como consecuencia la falta de recurso humano y financiero. Según lo expresa uno de los participantes:

"Todavía estamos funcionando de forma centralizada, por ejemplo en mi caso, presenté un proyecto de complementación a parteras por un valor de nueve millones de pesos y que pasa? Que en la dirección departamental un funcionario desde su escritorio sin conocer la problemática del municipio, sin ninguna proyección social, nos dice: doctor esa cantidad no se le puede asignar, a su municipio le corresponde trescientos mil pesos. También nos asignan una cantidad de dinero para el programa de lepra siendo que en esta zona lepra no hay. iEntonces! Recursos los hay pero están mal distribuidos. Esta situación afortunadamente ya empieza a cambiar con la descentralización administrativa porque ellos hacen una distribución de acuerdo a patologías sin tener en cuenta las necesidades locales. Por mucho que usted presente un plan estructurado, ellos son los que mandan".

- Con relación a gerencia de recurso humano se identificaron los siguientes factores:

- Gestión de recurso humano insuficiente por múltiples actividades administrativas y asistenciales a cargo de algunos directores.

"El nuevo marco de la seguridad social en Colombia e implementación de la ley 100 de 1993 hace que en este momento todas las actividades se centren en este proceso y que bajemos la guardia en la parte asistencial."

- Falta de continuidad e inexperiencia del personal encargado de la vigilancia. Los profesionales en servicio social obligatorio son los encargados de los programas pero al cumplir su periodo dejan el programa sin continuidad.

"En Corinto, por ejemplo, el médico rural implantó un programa de parto asistido, la partera asistía al centro hospital con su paciente, ella atendía el parto de bajo riesgo y en caso de alguna complicación allí estaba el médico. Pero cuándo esta persona terminó el año rural, se acabó el programa porque no correspondía a una política institucional y quien lo reemplaza llega con unas ideas totalmente diferentes." Auxiliar de Enfermería.

- El compromiso y motivación del personal médico y de enfermería con la vigilancia es bajo. Existen los protocolos de vigilancia, diagnóstico y manejo, los conocen pero no los aplican.

"Yo me pongo a mirar el COVE que funciona aquí, tiene la información, en muchas oportunidades podemos decir que la mortalidad está muy elevada y que vamos a disminuirla pero ¿dónde está el equipo, el médico, el enfermero y el auxiliar que va a participar en esa acción de respuesta inmediata? Siempre se plantea un ideal pero no hay respuesta en la parte operativa, entonces un COVE así no es operativo." Funcionario de la seccional norte.

"Pero por lo menos allá se reúnen y bien o mal funciona, pero en mi institución piensan que yo soy el encargado de hacer todo. Yo registro la información que me solicitan de la dirección pero nunca se analiza." Apunta una enfermera: "A las promotoras 
se las ha capacitado y por mas que se les insiste en la importancia que tiene la información acerca de las muertes de maternas o niños que ocurran en sus sitios de trabajo no lo hacen. ¿Quién cambia la mentalidad a una persona que lleva 20 años trabajando y próxima a jubilarse?" Auxiliar de estadística.

- Déficit y distribución anómala de funciones del personal en las regionales.

\begin{abstract}
"Si, yo si creo que el problema es de distribución de recurso humano pero no es solo ese: en algunos municipios han cancelado contratos entre ellos a las promotoras sociales, los cancelaron porque no hay con que pagarles, esas áreas en estos momentos están descubiertas, mientras que una promotora rural tiene a su cargo 150 familias y una urbana mas o menos 200, la promotora social no alcanzaba a tener ni cincuenta familias, entonces yo digo! La distribución del recurso humano no estaba bien. "Claro! No está bien" responde otra auxiliar de enfermería. "Porque nosotras tenemos recarga de trabajo Ah! Nooo... mientras en unos hospitales solo tienen que ver un programa nosotras tenemos a cargo tres o cuatro programas, lógico no está bien". Enfermero.
\end{abstract}

- Las relaciones inter-institucionales tanto administrativas corno asistenciales influyen directamente sobre aspectos de la mortalidad materna y perinatal.

- Falta de retroalimentación en la información sobre los partos atendidos en instituciones de salud.

"Yo quiero expresar el sentir nuestro respecto a la mortalidad materna y perinatal. Los aspectos relacionados con flujo de información, limpieza del dato, seguimiento y retroalimentación, si, representan un problema de vigilancia pero la falta de información y seguimiento son causas del mal funcionamiento del programa, en la Unidad Popayán no atendemos partos, estos se atienden en los hospitales: San José, Susana López, Comsalud (Una IPS local) e ISS . ¿y qué pasa con eso? No recibimos retroalimentación sobre la situación final del parto." Medico general.

- Falta de fiscalización y control de recursos de la Vigilancia Epidemiológica Departamental. Los entes encargados de fiscalizar los recursos y gerenciar la vigilancia epidemiológica departamental. (Vigilar y controlar los recursos financieros y el cumplimiento vigilancia en las direcciones locales, ARS e IPS) no lo hacen o su acción es limitada. Este hecho debilita el sistema a todo nivel. Ejemplo de ello son los problemas ya mencionados relacionados con el flujo de información, la prestación del servicios, la oportunidad y confiabilidad en el diagnóstico y la búsqueda de mercado sin control en materia de salud por parte de las ARS.

Por otra parte, es relevante resaltar la actitud positiva y el alto grado de compromiso demostrados por algunos grupos participantes:
"En Popayán, antes de 1996 no existían registros de la mortalidad materna y perinatal, ante el incremento de las tasas de mortalidad, según los datos presentados al inicio de esta sesión, nuestra preocupación fue tal, que en la Unidad Popayán, se trató de dar un gran empuje al trabajo interinstitucional en promoción y prevención, se han realizado talleres educativos de salud sexual y reproductiva, control prenatal, planificación familiar, y se implementó el modelo biosicosocial de atención a la gestante que ha servido muchísimo." "Yo considero que hemos mejorado en muchos aspectos, al menos en mi municipio (El Tambo) antes se tenía 4 promotoras, ahora hay 32, antes se hacía presencia en 11/210 veredas, ahora en 90/210, se realizó capacitación a parteras, se implementó una red de urgencias, se colocaron radioteléfonos y se aumentó el número de automotores. Esperamos con estas acciones facilitar las comunicaciones, el flujo de información, incrementar la atención del parto institucional y disminuir y/o controlar la mortalidad materna $\mathrm{y}$ perinatal".

\section{Factores Poblaciones}

- Aspectos económicos: Los funcionarios perciben que los usuarios tienen la creencia del alto costo de los partos institucionales y por lo tanto creen que la tendencia a la atención del parto por parteras es cada día mayor. También captan las dificultades para acceder a los sistemas de afiliación del régimen subsidiado y las deficientes relaciones interinstitucionales entre las empresas que realizan la afiliación y las que prestan el servicio. Así lo expresa uno de los participantes:

"Las empresas solidarias no quieren gastar recursos, las pacientes no firman la facturación de la atención que se les presta en el hospital, dicen: A mí me dijeron que no firmara esa factura porque me quitan plata de la que me corresponde por este carnet."

- Culturales y geográficas: Las diferencias culturales relacionadas con el control prenatal y la atención del parto hacen que las maternas busquen diferentes formas de atención de acuerdo a sus creencias; los funcionarios han encontrado mujeres que hacen el control prenatal en el hospital y a la hora del parto llaman la partera. También han percibido el rechazo de las maternas a la atención hospitalaria. Las madres se quejan de falta del trato considerado por parte del personal hospitalario.

"Nosotros estamos viendo por parte de las usuarias el rechazo a acudir al hospital, tenemos el caso de una paciente de la zona urbana con un mortinato de 23 semanas hizo una hipoxia fetal intrauterina y una hipertensión materna, en este caso la paciente nunca fue al hospital la información se consiguió posterior al hecho."

La accesibilidad geográfica, el estado de las vías, las rutas y horarios de los medios de transporte son factores 
que influyen en la atención de parto en las instituciones. En algunos municipios no hay carreteras sobre todo al sur del departamento; en otros el problema es el horario en las rutas. A estos factores, se suman los costos derivados del pasaje y el desplazamiento.

\section{Densidad de incidencia}

El segundo método de análisis es la Densidad de Incidencia la cual es definida como el número de veces que un tema es mencionado en cada grupo (El numerador es el número de veces que se menciona el tema y el denominador es el total de los grupos focales). La información así estructurada es de ayuda porque permite conocer la relativa importancia de temas identificados y expresados los participantes en cada uno de los grupos y aportes de los cuatro grupos en conjunto por los participantes. En esta ocasión también se estableció la diferenciación entre factores que afectan la Vigilancia Epidemiológica en el ámbito institucional y a escala poblacional. Como se puede observar en el Cuadro 1, hubo temas que se potencializaron y otros que emergieron.

\section{FACTORES INSTITUCIONALES Y POBLACIONES QUE AFECTAN EL PROCESO DE VIGILANCIA MATERNA Y PERINATAL}

NIVEL INSTITUCIONAL

\section{NIVEL POBLACIONAL}

\section{TEMAS QUE SE POTENCIALIZARON}

La falta de un sistema estructurado de vigilancia epidemiológica (Existencia de acciones para el control de la mortalidad materno-perinatal sin obedecer a un programa estructurado): $4 / 4$

Desconocimiento de los funcionarios del proceso de descentralización en salud, causó impacto negativo a nivel de las instituciones en los programas de vigilancia (Información, carnetización y prestación de los servicios, facturación): $4 / 4$

Falta de continuidad del personal profesional responsable del programa: $4 / 4$

Registro de datos de mortalidad sin análisis (La información del COVE no se utiliza en la planeación de acciones o programas preventivas): $3 / 4$

Ausencia de coordinación interinstitucional (Hospitales, ISS, ARS) en el sistema de vigilancia: $3 / 4$

Trastornos en la continuidad del control prenatal por diferentes entidades ofreciendo el servicio: $3 / 4$

\section{TEMAS QUE EMERGIERON}

Escasez de recursos económicos para el desarrollo del programa: $2 / 4$

Falta de información sobre Ia mortalidad maternoperinatal por parte de las empresas de medicina prepagada: $2 / 4$

Formación universitaria con déficit en aspectos de atención primaria, labor social y administración en salud: $2 / 4$
Conocimiento no sistemático de las diferencias culturales que puedan afectar la continuidad del control prenatal: $3 / 4$

Desconocimiento de la población acerca proceso de descentralización en salud, causó impacto negativo en los programas de vigilancia (Las usuarias del control prenatal no volvieron al hospital): $4 / 4$

Creencias erróneas de la comunidad acerca del costo del parto institucionalizado: $3 / 4$

Aspectos geográficos de acceso, rutas, horarios y costos de transporte que entorpecen la continuidad del control prenatal: $3 / 4$

Control prenatal irregular e incompleto en zona urbana: $3 / 4$
Dificultades económicas que desfavorecen la atención del parto institucionalizado y por ende el sistema de vigilancia: $1 / 4$

Percepción de frecuencia creciente de Embarazo en adolescentes: $1 / 4$

Diferencias culturales que no favorecen la comunicación con las maternas: $1 / 4$

Incremento de la atención del parto por parteras: 1/4 


\section{Discusion}

En términos generales, puede considerarse una apreciable coherencia en las discusiones entre las principales preocupaciones de los equipos de vigilancia ante el problema de la mortalidad materna y en la identificación de factores que afectan el proceso de la vigilancia y la formulación de propuestas para emprender el camino de las soluciones. Esto permite afirmar que existe entre los participantes conocimiento del problema. Siendo así, la pregunta sigue siendo: ¿Porque no se aplican las medidas existentes para superarlo?

La dependencia administrativa de la dirección departamental, la transición hacia un nuevo modelo de seguridad social en salud, la duplicidad de funciones administrativas y asistenciales de algunos funcionarios, análisis incompleto de las muertes ocurridas que generalmente no conducía a intervenciones oportunas y adecuadas, la falta de importancia dada a este problema por parte de algunos directores, la actual forma de contratación de prestación servicios de salud, el desconocimiento tanto del personal de salud como de la comunidad del funcionamiento del nuevo sistema de seguridad social que produjo rechazo y disminución en la demanda de atención institucional del control prenatal y de1 parto son hechos claves que favorecen y perpetúan la presencia del problema más no lo justifican. Podríamos establecer la hipótesis que la falta de estructuración de un sistema de vigilancia en medio del cambio de modelo de salud y seguridad social produce una actitud en las personas lo que hace que el problema se perpetúe.

Por otra parte la escasez de personal o la distribución del mísmo en la ínstitución sin criterios claros se combinaba con debilidades en el conocimiento del contexto social y cultural y por lo tanto la aproximación comunicativa con las mujeres embarazadas y en edad reproductiva era difícil, principalmente en los municipios cuya composición étnica era variada (Guambianos, Paeces y/o comunidades afrocolombianas). Estos hechos ya se han relacionado con el incremento de mortalidad materna (13).

Se sugiere la constitución de una política estructurada de la Dirección Departamental de Salud en la vigilancia epidemiológica de la mortalidad materno-perinatal que esté inmersa en los retos de un concepto ampliado de salud materna (14) y que tenga en cuenta aspectos como: formación de recurso humano basado en un programa de capacitación continua (La capacitación debería incluir formación socio-antropológica), apoyo a la gestión de los sistemas de vigilancia como proyectos con asignaciones presupuestales para recurso humano y logístico, continuidad del personal profesional responsable en el programa, optimización de la comunicación interinstitucional, fortalecimiento del modelo biosicosocial, estructuración del sistema de informacion y registro en las historias clínicas prenatales, elaboración y difusión de protocolos del sistema de vigilancia de la mortalidad materna-perinatal, y fortalecimiento operativo y decisorio de los COVE. Es posible que una política estructurada de esta manera ayude a revertir la tendencia ascendente de la mortalidad materna en la región.

\section{Agradecimientos}

A John Arcila, Carlos Caicedo, Claudia Díaz, Jaime Martínez, John Reyes, Yamil Sandoval, alumnos de la facultad de Ciencias de la Salud, quienes colaboraron en la conformación y conducción de los grupos focales.

\section{BIBLIOGRAFIA}

1. Mora G, Yunes J, Mortalidad materna: una tragedia ignorada. En género, mujer y salud en las Américas, publicación científica No. 541, OPS, Washington, 1993

2. Plan de acción regional para la reducción de la mortalidad materna en las Américas, Bol Of Sanit Panam 110(5), 1991.

3. Echeverry ML, Loaíza 1B, Vélez LM Vigilancia epidemiológica en mortalidad materna y perinatal en Antioquia, Boletín epidemiológico de Antioquia, 1993; 18: 40-46.

4. Dirección seccional de salud de Antioquia, Oficina de Epidemiología, Protocolos de Vigilancia epidemiológica, 4a. Ed. Medellín, 1996.

5. Tapia R. La importancia de la vigilancia epidemiológica en los servicios de medicina preventiva, Epidemiología, Sistema nacional de Vigilancia epidemiológica, México, 13; $32 . \quad$ Agosto 1996.

6. Dirección Seccional de salud del Cauca. Informes sobre vigilancia epidemiológica de la mortalidad rnaterno-perinatal en municipios del Cauca. Popayán, 1997

7. Bender D, Ewbank D. The focus group as a tool for health reseach: issues in design and analysis, Health Transition Review vol $4 \mathrm{~N}^{\circ} 1$, 1994.
8. Vong Ek, P Focus group technique En Youdummen-Attig B, Attar, $\mathrm{G}$, Boonchalansky V. A field manual on selected qualitative researh methods, Mahidol University, Thailandia, 1989.

9. Wilms D Johnson N Focus groups. En: Essential in Qualitive researh. A note-book for the field. Documento interno no publicado. McMaster University, Canadá

10. Maholtra NK, Investigación de Mercados, Simon\&Schuster Co. México, 1997, cap. 5.

11. Youdummen-Attig (1989) Cap. 5. Data analysis and report writing. En Youdummen-Attig, A field manual of selected qualitative researh methods, Documento interno no publicado, Mahidol Unversity, Thailandia

12. Salvador J, De la Luna E, Reid A, Rivera L. Un enfoque biosicosocial de la enfermedad hipertensiva aguda del embarazo, Salud Pública Mex 1989; 31: 757-762

13. Blanco Muñoz J. y cols, Mortalidad materna en el área de Huichol del estado de Jalisco, México, Salud Pública Mex 1994; 36: $263-$ 268.

14. Villegas C, Luna J. La salud materna: un gran reto para Colombia, Revista de Salud Pública, vol 1, $\mathrm{N}^{\circ}$ 1, Facultad de Medicina, Universidad Nacional de Colombia, Bogotá, Marzo, 1999. 